\title{
MEASUREMENT AND SIMULATION OF SOURCE-GENERATED HALOS IN THE UNIVERSITY OF MARYLAND ELECTRON RING (UMER)*
}

\author{
I. Haber ${ }^{\#}$, S. Bernal, R. A. Kishek, P. G. O'Shea, C. Papadopoulos, M. Reiser, \\ R. B. Feldman, D. Stratakis, M. Walter UMD, College Park, Maryland, 20742, U.S.A. \\ J.-L. Vay, LBNL, Berkeley, California, U.S.A. \\ A. Friedman, D. P. Grote, LLNL, Livermore, California U.S.A..
}

\section{Abstract}

One of the areas of fundamental beam physics that have served as the rationale for recent research on UMER is the study of the generation and evolution of beam halos. Recent experiments and simulations have identified imperfections in the source geometry, particularly in the region near the emitter edge, as a significant potential source of halo particles.

The edge-generated halo particles, both in the experiments and the simulations are found to pass through the center of the beam a short distance downstream of the anode plane. Understanding the detailed evolution of these particle orbits is therefore important to designing any aperture to remove the beam halo.

\section{INTRODUCTION}

The UMER experiment is an electron ring designed to study, on a scaled basis, the basic physics of a highly space-charge-dominated beam.[1]. One area of the physics studied on UMER, and that is of interest to a number of accelerator systems, is the dynamics of halo generation, as well as possible mitigation techniques. Halos can be generated by a number of dynamical processes as the beam propagates. However, a halo can also be generated in the source region. Furthermore, halo generation by space charge in the source region can be important even in accelerators where space charge is not significant for most of the accelerator length, since the beam in these accelerators is often dominated by space charge when born, and a halo generated early in the beam lifetime can, in general, persist as the beam is accelerated.

\section{THE UMER GRIDDED ELECTRON GUN}

The UMER gridded electron gun is shown schematically in Fig. 1. The cathode grid is used for temporal control of the beam pulse, at the expense of complicating the initial beam distribution. Under some conditions, for example, a hollowing of the velocity space as well as well as virtual cathode oscillations downstream of the cathode grid can result.[2] A rotating aperture plate, at the plane shown, can be used to insert different apertures approximately $1 \mathrm{~cm}$ downstream of the gridded anode.

\footnotetext{
*Work supported by his work is supported by the US DOE under contract Nos. DE-FG02-02ER54672 and DE-FG02-94ER40855 (UMD), and DE-AC02-05CH11231 (LBNL) and W-7405-ENG-48 (LLNL)

"haber@umd.edu
}

Dynamic halo generation is one of the areas of research planned for UMER. However, a substantial beam halo, as shown in the photographic image in Fig. 2, was observed on the phosphor screen approximately $30 \mathrm{~cm}$ downstream of an aperture, intercepting approximately $80 \%$ of the beam current. An initial beam halo, particularly if not well understand, obviously complicates the experimental study of downstream mechanisms for halo generation. Also, because halo generation is often a phase space phenomenon, removal of a halo by using apertures to scrape only those halo particles that appear in configuration space at the aperture plane, can be difficult. It is therefore important to determine the cause of the halo so that mitigation can be performed at the cause rather than trying to eliminate the halo by scraping downstream.

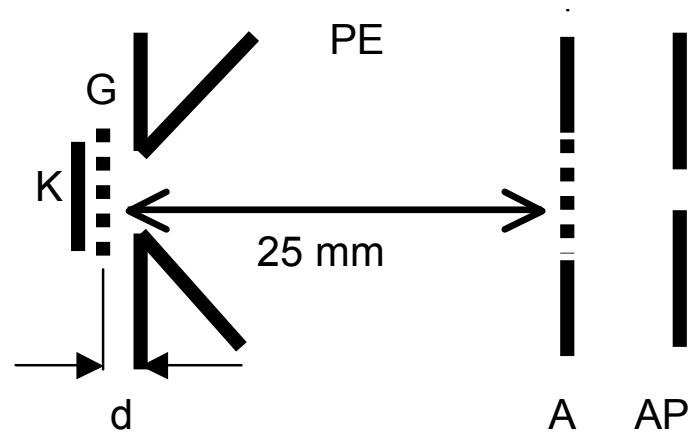

Fig.1. Schematic diagram of the UMER gun showing the relative positions of the cathode $(K)$, cathode grid $(G)$, Pierce electrode (PE), gridded anode $(A)$, and aperture plate (AP). The space between the cathode grid and the back of the Pierce cone is designated as $d$.

Under the current operating conditions in UMER, the difficulty usually encountered in halo scraping is accentuated by the observation that inserting the various apertures that are available on the aperture wheel immediately downstream of the anode had little observable effect on the halo. This was interpreted as evidence that the particles in the halo pass close to the center of the beam at the aperture plane. 


\section{SIMULATION OF THE UMER SOURCE}

Figure 3 is a particle plot of the r-r' phase space at the aperture plane showing the predicted beam behavior for the nominal gun design that has a $\mathrm{d}=0.1 \mathrm{~mm}$ gap between the back surface of the Pierce cone and the cathode grid. The simulation was performed using the RZ module of the WARP electrostatic PIC code and made use of the mesh refinement capability,[3] employing an axial resolution of $0.075 \mathrm{~mm}$ and transverse resolution of 0.125 $\mathrm{mm}$ in the vicinity of the cathode.

A common issue in gun design is created by the necessity to provide a separation between the hot cathode structure and the remainder of the gun, particularly the Pierce electrode, to provide a degree of thermal insulation. In the UMER case this hot structure includes the cathode grid. It has been known for some time [4,5] that the details of this separation can cause a substantial modification to the particle orbits in the vicinity of the edge of the emitter. In UMER, as in many gun designs, this distance is difficult to control and measure, and is therefore not precisely known.

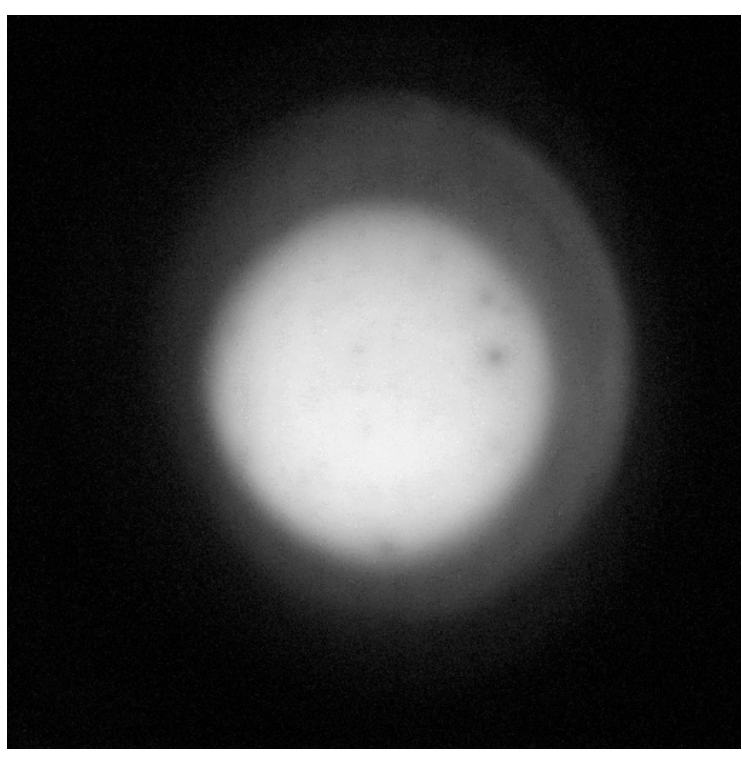

Fig 2. Phosphor screen image of the UMER beam showing halo particles visible approximately $30 \mathrm{~cm}$ downstream of the aperture plate. The beam has passed through a $3 \mathrm{~mm}$ diameter centered aperture that passes approximately $20 \%$ of the beam current, followed by a focusing solenoid magnet. Note that the small spots are damage spots on the phosphor.

Figure 4 is an r-r' phase space plot at the aperture plane showing the consequence of increasing the distance between the cathode grid and the back of the Pierce cone from the nominal $\mathrm{d}=0.1 \mathrm{~mm}$ to $0.5 \mathrm{~mm}$. The creation of a halo of particles, distinct from the main distribution can be seen near the beam center.

The mechanism that creates the particles responsible for the halo can be seen to result from the characteristics of the electric field in the vicinity of the inner edge of the Pierce cone. Figure 5 is a plot of the equipotential lines in the vicinity of the Pierce cone edge. Since the electric field is proportional to the gradient of these lines the gap between the Pierce cone and the cathode grid results in an inward directed field in the vicinity of the gap that accelerates particles toward the beam center. For the parameters of the UMER gun structure these particles reach the vicinity of the beam center at the aperture plane.

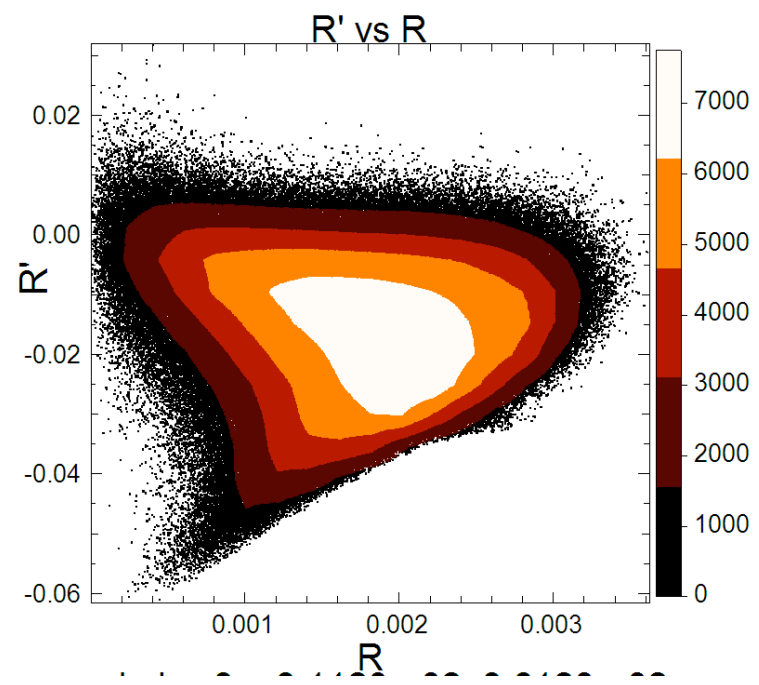

Fig. 3 WARP-predicted $r-r^{\prime}$ phase space at the aperture plane for the nominal $\mathrm{d}=0.1 \mathrm{~mm}$ spacing between the cathode grid and the back of the Pierce cone.

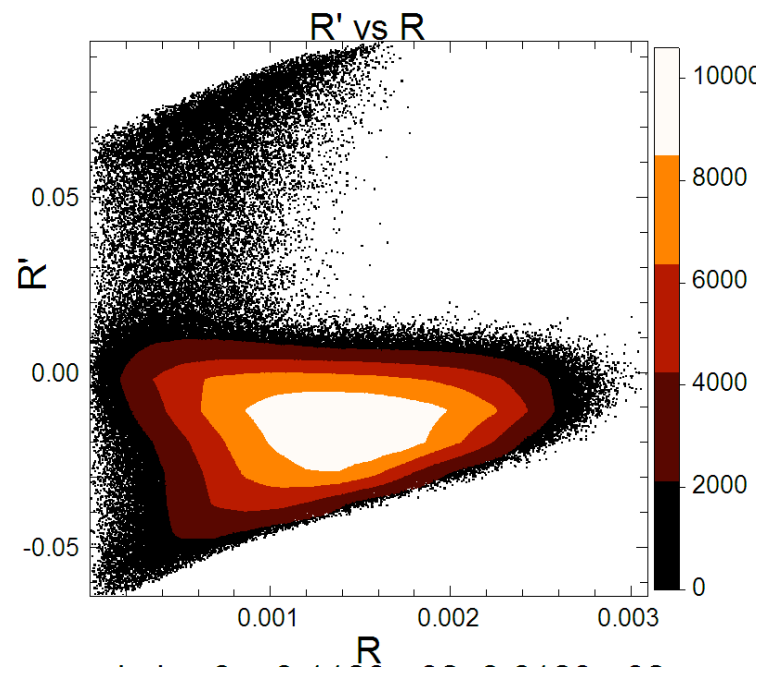

Fig.4. WARP-predicted $r-r^{\prime}$ phase space at the aperture plane when the spacing between the cathode grid and the back of the Pierce cone is increased to $\mathrm{d}=0.5 \mathrm{~mm}$. Note that the extra particles outside of the main body of the distribution are near the beam center. 


\section{HALO MITIGATION}

The mechanism that causes the halo in the UMER gun suggests that a simple way to substantially reduce this effect is to modify the geometry so that there are no beam particles in the region of the Pierce cone edge. Figure 6 is the phase space plot that results from increasing the inner diameter of the anode grid from $8 \mathrm{~mm}$ to $10 \mathrm{~mm}$. Since the diameter of the cathode emitter is $8.5 \mathrm{~mm}$, the edge particles are no longer strongly influenced by the field distortion at the edge of the Pierce cone. It should be noted that the cathode grid, whose diameter is somewhat larger than even the enlarged Pierce cone aperture, accelerates the particles emerging from the cathode surface so that they are not expected to spread substantially in the radial direction before passing through the Pierce-cone aperture. Furthermore, simulations performed for different values of the spacing, $d$, between the cathode grid and the back of the Pierce cone show that the phase space distribution is relatively insensitive to this dimension now that the Pierce cone aperture has been enlarged.

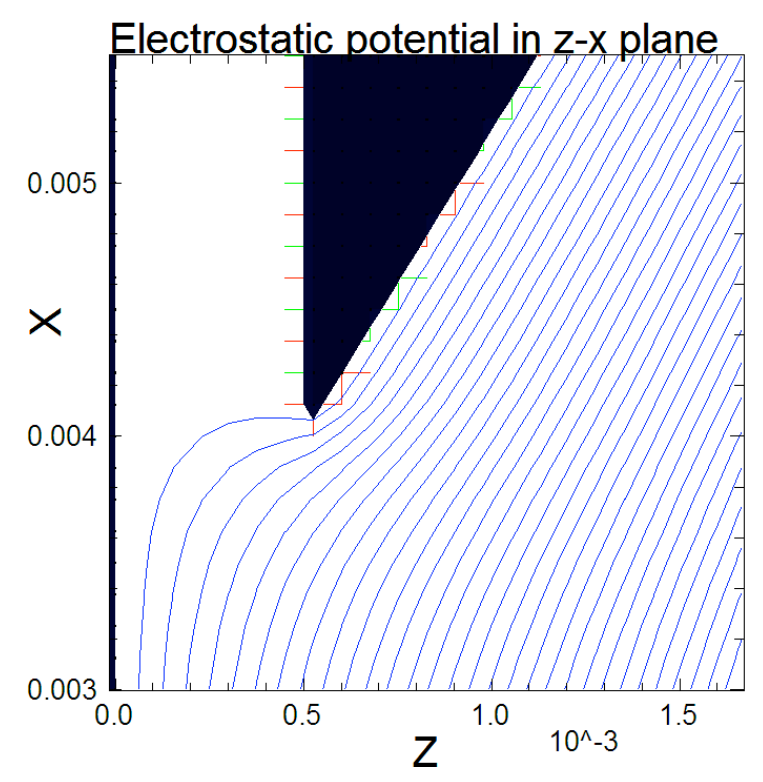

Fig 5. Expanded-scale plot of equipotential lines showing distortion of those lines in the vicinity of the edge of the Pierce cone. Since the electric field is proportional to the gradient of the potential, an inward directed field is present that accelerates particles at the beam edge toward the beam center.

Though the simulations predict that substantially improved gun characteristics will be obtained by enlarging the Pierce-cone aperture, actual performance may vary. The actually-constructed behavior is subject to machining errors as well as to small misalignments. For example, it can be seen in Fig. 2 that the main beam distribution is slightly off-centered relative to the halo. This is thought to result primarily from a slight lack of parallelism between the emitter surface and the anode.

Understanding the actual consequences of enlarging the Pierce-cone aperture must await experiment. It is therefore planned to replace the Pierce cone electrode in the future when the gun is brought to atmospheric pressure.

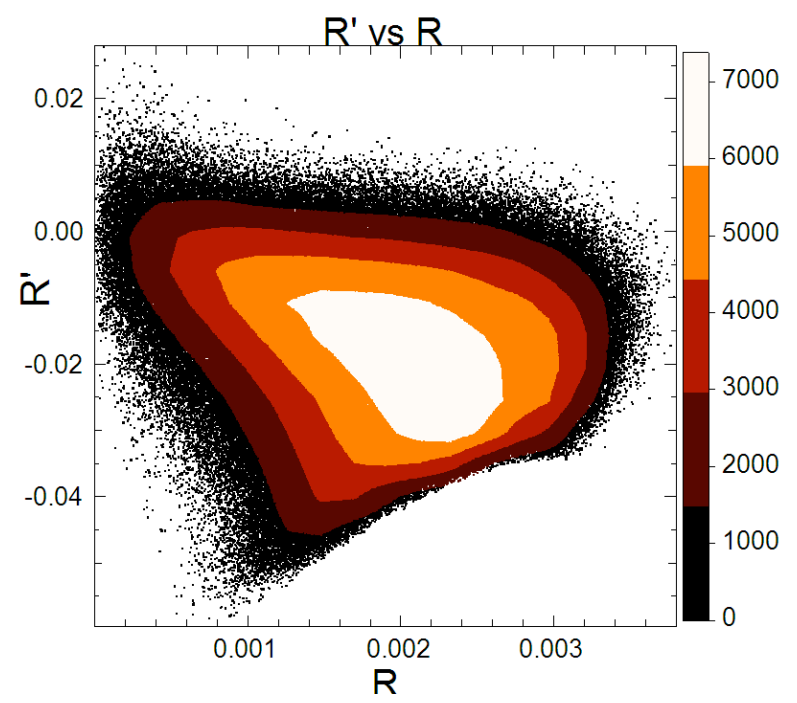

Fig.6. WARP-predicted $r-r^{\prime}$ phase space at the plane of the aperture plate showing the substantial reduction in halo when the inner aperture of the Pierce cone is enlarged from a radius of $4 \mathrm{~mm}$ to $5 \mathrm{~mm}$. The distance between the cathode grid and the back of the Pierce cone is $0.5 \mathrm{~mm}$ as in Fig. 4 .

\section{REFERENCES}

[1] R. A. Kishek, et. al. Paper TUZBAB03, Proceedings, $\mathrm{PAC} 07$

[2] I. Haber, D. Feldman, R. Fiorito, A. Friedman, D. P. Grote, R. A. Kishek, B. Quinn, M. Reiser, J. Rodgers, P. G. O'Shea, D. Stratakis, K. Tian, J. -L. Vay, M. Walter NIMA 577, 150 (2007).

[3] J.-L. Vay, P. Colella, J.W. Kwan P. McCorquodale, D.B. Serifini, A. Friedman, D.P. Grote, G. Westenskow, J.-C. Adam, A. Heron, and I. Haber, Physics of Plasmas 11(5), 2928 (2004).

[4] W. B. Herrmannsfeldt, private communication.

[5] F. Hegeler, M. Friedman, M. C. Meyers, J. D. Sethian, S. B. Swanekamp, Phys. Plasmas, 9(10), 4309. (2002). 